\title{
Formation and Pattern of Hexagonal Pyramid and Triangular Etch Pits and Relationship to Dislocation in Deformed Nickel Based Super Alloys
}

\author{
Aezeden Mohamed \\ Faculty of Engineering, University Manitoba, Winnipeg, Canada, R3T 5V6
}

\begin{abstract}
Two nickel-based super alloys, alloy I and alloy II, were annealed through heat treating and were heavily deformed under a dynamic load. After sectioning and sample preparation, both alloys were chemically etched with modified Kalling's II reagent. Microscopic observation revealed hexagonal pyramid and triangular etch pit formation. Results were compared to as received both alloys before loading. Possible explanations for etch pit formation are presented.
\end{abstract}

Keywords: - Dislocation, Etch Pits, Hexagonal Pyramid, Triangular.

\section{INTRODUCTION}

One of the means for direct observation of dislocations is to study selectively etched surfaces of the material. Several investigators [1-3] have reported on such studies. The successful use of etch pits to study dislocations in nickel-based super alloys depends appreciably on the etchant and the experimental techniques employed. Other work on etch pits made use of Burgers vectors to map the lattice distortion of the dislocations as well as determining crystallographic relationships between dislocation directions and etch pits [4].

Another study on etch pits in biotites and found the development of etch pits in the (001) plane was influenced by dislocations and lattice defects [5]. This phenomenon could lead to etch pits in the (001) plane developing in an unpredictable manner for biotites in both triangular and hexagonal forms. On the other hand, report showed that the development of the etch pit with depth may depend on the mica polytype; the different polytypes may exist in the same mica [6].

Further work on etch pits were found in the (101) and (100) planes [7]. Two types of etch pits were revealed in the form of triangular etch pits on pyramidal faces and parallelogram-shaped etch pits on prismatic faces. The latter may be related to line defects like dislocations.

The purpose of this work to show the value of Kalling's II etchant or to show that while this etchant does provide a strong contrast and detail, the results are difficult to interpret because the etch pits are intended to identify areas of dislocations but not precipitates. When the etch pits may be caused by both, it reduces the values of the etchant for dislocation studies.

\section{EXPERIMENTAL PROCEDURE}

As-received two nickel based super alloys I and II, as show in Table 1 and Table 2 were heat treated at $1000^{\circ} \mathrm{C}$ for 1 hour and subsequently air-cooled and were heavily deformed under tension compression at a constant frequency $10 \mathrm{~Hz}$ cyclic loading and prepared for microscopic observations.

An EDM machine was used for cross sectioning to produce several samples from each alloy which were then mounted in bakelite by using a Buehler mounting press. The mounted sections were ground and polished to a $1 \mu \mathrm{m}$ finish, after which they were chemically etched in modified Kalling's II reagent etchant. These etchants enhance contrast of grain boundaries for microscopic observations and provide more details of the etch pits in the grain structures.

Samples were etched using a swabbing method for approximately 50 seconds. Below 50 seconds, the etchant was not able to reveal details of etch pits and above 50 seconds the etchant produced a dark film on the surface. After the required time of etching, the samples were thoroughly cleaned with water and then flushed with ethanol and dried with hot air.

SEM micrographs of these samples were taken immediately after etching. Detailed microscopic observation and chemical analysis by semi-quantitative and spectroscopy analyses was performed using a JEOL 5900 scanning electron microscope (SEM) equipped with an Oxford (Oxford Instruments, Oxford, United Kingdom) ultra-thin window energy dispersive spectrometer (EDS) as shown in Figure 1 and Tables 1 and 2 . 


\section{RESULTS AND DISCUSSIONS}

SEM micrographs showed no evidence of etch pits for as received alloy I and alloy II as shown in Figure 1 (a) and Figure 1 (b). The chemical analyses obtained by EDS for alloy I and alloy II are shown in Figures 1 (c) and 1 (d), respectively. It is noted that alloy I shows massive and homogenously dispersed precipitates as compared to alloy II which had none. These precipitates are obstacles and retard grain growth in the case of alloy I, whereas alloy II, without precipitates, shows grain growth and a larger grain size. These precipitates are generally referred to as MC carbides $[8,9]$. Both alloys were prepared and etched under the same conditions and underwent exactly the same heat treatment.
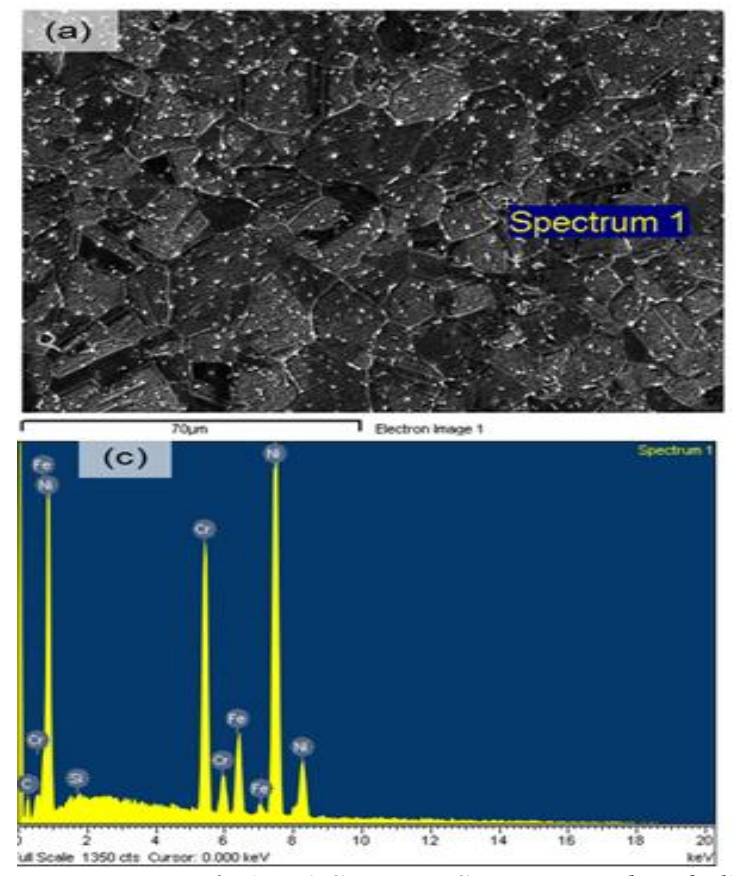

Figure 1: $(a, c)$ SEM-EDS micrographs of alloy I, $(b, d)$ SEM-EDS micrographs of alloy II.
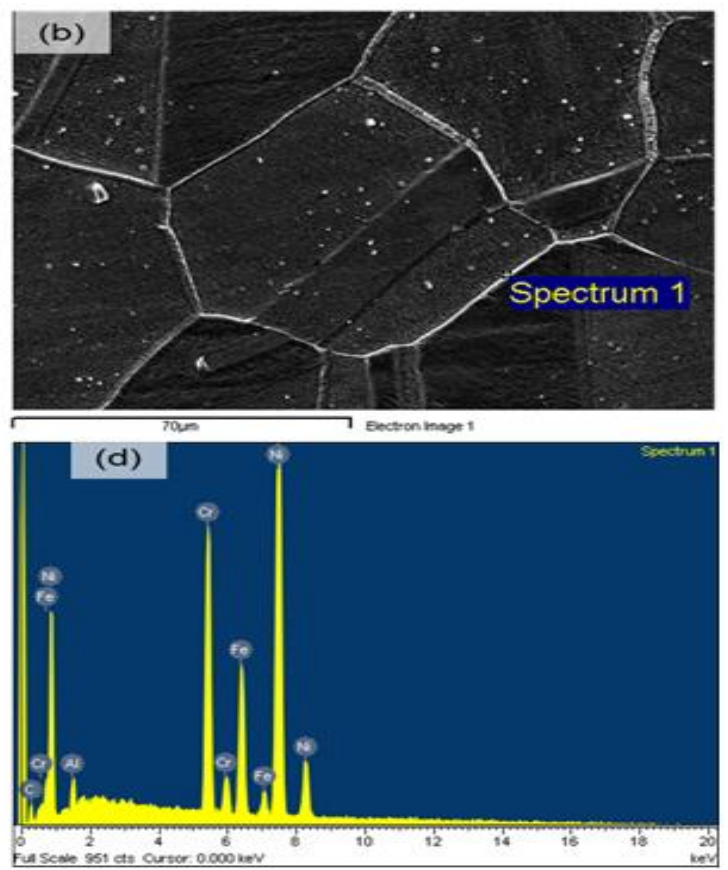

Table 1 Chemical analysis of alloy $I$

\begin{tabular}{|c|c|c|c|c|c|}
\hline \multicolumn{6}{|c|}{ Table 1: Chemical analysis of alloy I } \\
\hline Element & App & Intensity & Weight \% & Weight \% & Atomic \% \\
\hline & Conc. & Corrn. & & Sigma & \\
\hline C K & 2.92 & 0.3734 & 6.35 & 0.52 & 24.23 \\
\hline Si K & 0.22 & 0.5773 & 0.30 & 0.10 & 0.49 \\
\hline Cr K & 26.22 & 1.0352 & 20.58 & 0.34 & 18.14 \\
\hline Fe K & 10.31 & 1.0482 & 7.99 & 0.27 & 6.56 \\
\hline Ni K & 75.87 & 0.9514 & 64.78 & 0.55 & 50.57 \\
\hline & & & & & \\
\hline Totals & & & 100.00 & & \\
\hline
\end{tabular}

Table 2 Chemical analysis of alloy II

\begin{tabular}{|c|c|c|c|c|c|}
\hline Element & App & Intensity & Weight \% & Weight \% & Atomic \% \\
\hline & Conc. & Corrn. & & Sigma & \\
\hline $\mathrm{CK}$ & 2.41 & 0.3696 & 4.89 & 0.55 & 19.28 \\
\hline Al K & 0.91 & 0.4548 & 1.50 & 0.14 & 2.62 \\
\hline Cr K & 27.13 & 1.0408 & 19.59 & 0.40 & 17.83 \\
\hline Fe K & 19.59 & 1.0383 & 14.18 & 0.40 & 12.02 \\
\hline $\mathrm{Ni} \mathrm{K}$ & 75.43 & 0.9472 & 59.84 & 0.64 & 48.25 \\
\hline Totals & & & 100.00 & & \\
\hline
\end{tabular}


The annealed alloy I indicating hexagonal pyramid, triangular etch pits within grains and along grain boundaries is shown in Figure 2.
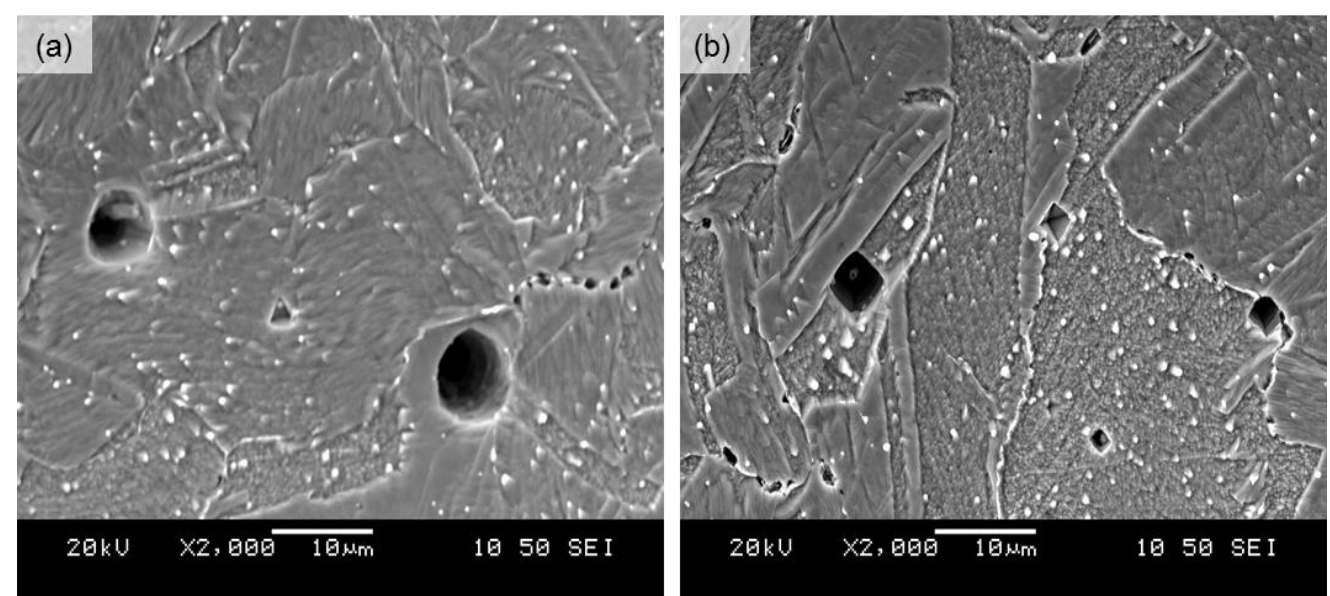

Figure 2. SEM micrographs show annealed alloy I with, (a) triangle shaped etch pits large and small.

By contrast, alloy II shown in the SEM micrographs in Figure 3, has massive etch triangular pits, and these pits were arranged in lines within the grains. No triangular etch pits were observed within the grain boundaries. Furthermore, some pits grow in lateral dimensions and in depth, as shown in Figure 3 and Figure 4.
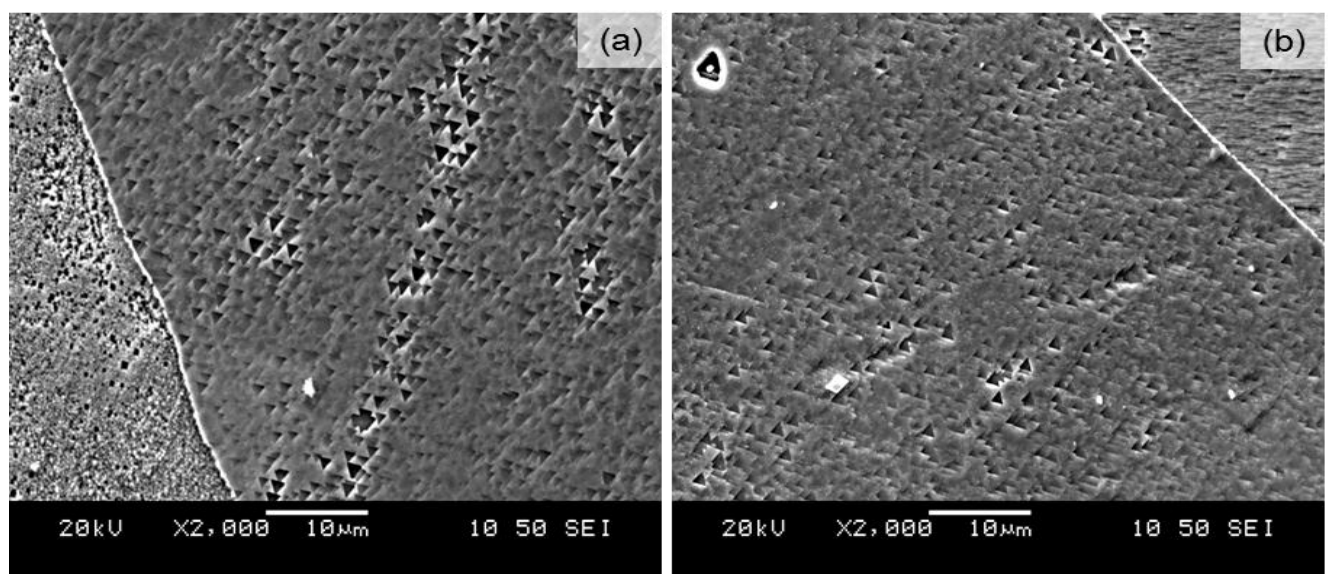

Figure 3. SEM micrographs of annealed alloy II showing massive triangular shaped etch pit in both (a) and (b).

Also, the SEM micrograph of alloy II shows triangular etches pits (Figure 4) (a) and square etch pits (b).
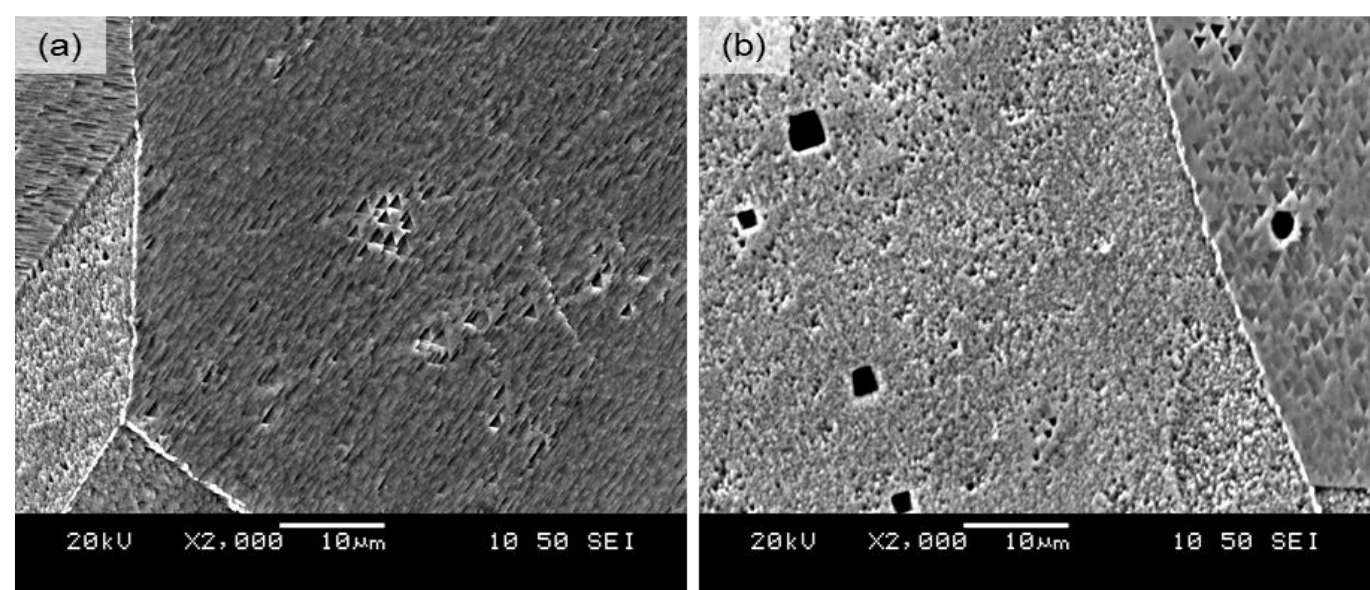

Figure 4. SEM micrographs of annealed alloy II, (a) showing triangular etch pit and $(b)$ triangular and square pits can be observed on one of the grains. 


\section{DISCUSSION}

Etching techniques have been successfully employed by several investigators for determining the configuration of dislocations within materials [10-13]. Very clear and detailed etch pits in alloys I and II have also been produced in this study with modified Kalling's II reagent etchant.

The etch pit formations are the results of imperfections occurring in the nickel-based super alloys. It may be easier to study etch pit formations and causes by using single crystals and in pure metals rather than in polycrystalline materials as in this current study. A number of studies of etch pits in single crystal or in pure metals $[1,4]$ have been reported. However, these studies were not in agreement as to how pits formed and why they formed. From the currently study, the causes cannot be unequivocally established but may be due to dislocation, precipitates, and may be due to specific heat treatment and the specific chemical etchant or a combination of all.

When dislocation lines intersect the surface of the annealed and heavily deformed alloy I and alloy II, the associated strain field locally increases the relative susceptibility of these two alloys to the chemical acidic etching and etch pits formations created. Because both alloys were heavily deformed and repeatedly re-etched by swabbing, a series of etch pits can be created which effectively trace the movement of the dislocations. Scanning electron microscopy is not appropriate for the direct observation and study of dislocations. Rather, transmission electron microscopy is the appropriate tool.

After chemical etching is applied on the surface of these two alloys, small pits are formed where the etching solution preferentially attacks the sample surface around the dislocations intercepting this surface and resulting from the heavily deformed and highly strained state from cyclic deformation. Thus, the SEM micrographs indicate series of etch pits formations at which dislocations intercept the sample surface. In this way, dislocations in nickel based super alloy can be observed indirectly when a sample is etched and placed under the scanning electron microscope. These series of etch pits can act in the same way as high dislocation densities when alloys are heavily deformed as shown in Figure 2, Figure 3 and Figure 4.

The geometry and morphology of etch pit varies even in same sample, etch pit has no relation to dislocation, and etch pits might be related to screw dislocations. A number of studies made the general observation of hexagonal shaped etch pits and dislocations, but not eentirely convincing evidence has been presented yet[14]. Further, crystal orientation can be determined by the shape and pattern of the etch pits associated with the dislocations; the current work did not go to this step which is planned for the next stage of study. The other explanation for the formation of etch pits is the chemical etchant in contact with the surface of the alloy samples. If the modified Kallings II reagent has a tendency to reveal precipitates this means the precipitates are points of easy attack by the etchant. If this is the case, the etch pits would be formed by nothing more than the leaching out of the precipitates and the leaving behind of voids whose geometry reflects that of the precipitates. The question can then be raised as to why this does not apply in the case of as received alloy I and alloy II as shown in Figure 1 (a) and Figure 1 (b) which revealed no hexagonal pyramid and triangular etch pit formation.

The results obtained may support this explanation of the formation of the etch pits and it was proposed that the pits were the result of a combination of both dislocation sites and the interaction of dislocations sites and precipitates, since precipitates can act as a source of dislocation formation.

This explanation would account for the great depth and small cross section of the etch pits which give the impression that the density of etch pits is related to the quantity of precipitates and hence to the production of a high dislocation density. The latter depends in some way on the crystal orientation of the alloy itself.

\section{CONCLUSION}

This brief microscopic study on heavily deformed nickel-based super alloy revealed hexagonal pyramid and triangular etch pit formation. The formation of etch pits may be a result of dislocation and precipitates interaction where they intercept on the sample surface. It is anticipated that this microscopic study will motivate further research on the unanswered questions about these etch pits.

\section{REFERENCES}

[1]. M. S, Joshi, and A. S. Vagh, A selective etch method for studying structural defects in cultured quartz, Crystallography, 12, 1967, 656-659.

[2]. A. Sagar, and J. W. Faust, Discrepancies from a perfect match in the etch patterns on the opposite cleavage faces in $\mathrm{Bi}_{2} \mathrm{Te}_{3}$, Applied Physics, 38, 1967, 2240-2243.

[3]. E. V. Tsinzerling, and Z. A. Mironova, Revealing of dislocations in quartz by selective etch methods, Crystallography, 8, 1963, 117-120..

[4]. Y. C. Akgoz, and G. A. Saunders, Dislocation etch pits and growth of arsenic-antimony single crystals, Journal of Materials Science, 6, 1971, 395-402. 
[5]. J. A. Medina, M. Morante, and S. Leguev, Some observations on the relationship between etch-pit form and structure in biotite, Clay Minerals, 20,1985, 263-271.

[6]. J. D. Martin Ramos and M. Rodriguez Gallego, Coexistencia de moscovitas 2M1en gneises de la unidad de la Caldera (Cordillera), Est. Geology. 36, 1980, 201-204.

[7]. S. Javidi, H. Faripour, M. Esmaeil Nia, K.F. Sepehri, and N. Ali Akbari, Development of a KDP crystal growth system based on TRM and characterization of the grown crystals, Semiconductor Physics, Quantum Electronics and Optoelectronics, 11, 2008, 248-251.

[8]. Y. Kawahito, T. Terajima, H. Kimura, T. Kuroda, K. Nakata, S. Katayama and A. Inoue, High-power fiber laser welding and its application to metallic glass $\mathrm{Zr}_{55} \mathrm{Al}_{10} \mathrm{Ni}_{5} \mathrm{Cu}_{30}$, Materials Science and Engineering B, Solid-State Materials for Advanced, Technology, 148(1-3), 2008, 105-109.

[9]. M. Donachie and S. Donachie, Superalloys: A Technical Guide, $2^{\text {nd }}$ Ed., OH: ASM International, pp. 3537, 2002.

[10]. V. Hari Babu, and K. G. Bansigir, Branching and bending of dislocation in $\mathrm{NaCl}$ crystals, Journal of Applied Physics, 40, 1969, 4306-4313.

[11]. S. Amelinckx, The direct observation of dislocation nets in rock salt single crystals, Philosophical Magazine I, 1956, 269-290.

[12]. I. V. K. Bhagavan Raju, T. Bhima Sankaram and K. G. Bansigir, Helical dislocations in as grown sodium chloride crystals, Journal of Applied Physics, 40, 1969, 4668-4669.

[13]. W. Bontinck, Climb phenomena in synthetic fluoride crystals, Philosophical Magazine, 2, 1957, 561-567.

[14]. A. Shintani, and S. Minagawa, Etching of GaN using phosphoric acid, Journal of Electrochemical Society, Solid-State Science and Technology, 123(5), 1976, 706-123. 